\title{
Explorando os teoremas de Menelaus e Ceva em questões de olimpíadas de matemática
}

\author{
Jhonata Avelar dos Santos ${ }^{1}$ (D)
}

\author{
Pedro Victor S. Freitas ${ }^{2}$ (D) \\ Thiago Yukio Tanaka ${ }^{4}$ (D)
}

Gilson S. Ferreira Jr. ${ }^{3}$ (D)

\section{Resumo}

Neste trabalho discutiremos sobre os Teoremas de Menelaus e de Ceva, que são dois resultados relacionados, respectivamente, com problemas envolvendo colinearidade entre pontos e concorrência de retas ou segmentos. Mais especificamente, exploraremos o uso desses dois teoremas na busca por soluções de problemas em Olimpíadas de Matemática nacionais e internacionais. Além do uso desses resultados no contexto olímpico, outro objetivo é contribuir com a formação e despertar o interesse de professores(as) e alunos(as) em competições olímpicas de Matemática, de forma que possa ser utilizado como material de apoio para o treinamento pelos docentes e um material de pesquisa para os discentes interessados.

Palavras-chave: Teorema de Ceva; Teorema de Menelaus; Olimpíadas de Matemática; Geometria.

\begin{abstract}
In this paper, we will discuss the Menelaus and Ceva Theorems, which are two results related, respectively, with problems involving collinearity between points and concurring lines or segments. More specifically, we will explore the use of these two theorems in the search for solutions to national and international Mathematical Olympiad problems. In addition to the use of these results in the Olympic context, another goal of is to contribute to the training and to arouse the interest of teachers and students in Olympic mathematical competitions, so that it can be used as support material for training by teachers and research material for interested students.
\end{abstract}

Keywords: Ceva's Theorem; Menelaus's Theorem; Math Olympiads; Geometry

\section{Introdução}

Os Teoremas de Menelaus e de Ceva são dois teoremas de épocas diferentes, mas intimamente ligados. Antes de começar a falar sobre os teoremas, vamos conhecer um pouco da história desses dois matemáticos e, consequentemente, de seus teoremas. Menelaus nasceu em 70 d.C. na cidade de Alexandria, no norte do Egito. Ele trouxe importantes contribuições na área de Geometria e dentre

\footnotetext{
${ }^{1}$ Discente do curso de graduação em Matemática da UFRPE.

${ }^{2}$ Discente do curso de graduação em Matemática da UFRPE.

${ }^{3}$ Docente do Departamento de Matemática da UFRPE.

${ }^{4}$ Docente do Departamento de Matemática da UFRPE.
} 
suas publicações destacam-se a obra Sphaerica [12], que trata de superfícies esféricas, e um teorema que leva o seu nome, que será um dos objetos de estudo deste trabalho. O Teorema de Menelaus lida com triângulos e proporções que uma reta gera ao intersectar seus lados e/ou prolongamentos de lados. Aproximadamente 15 séculos depois do nascimento de Menelaus, nasce na Itália, Giovanni Ceva. Estudante promissor, tornou-se matemático, físico e engenheiro hidráulico [12]. Em 1678 publicou o livro De lineis rectis se invicem secantibus, statica constructio, onde demonstrou um resultado que mais tarde ficou conhecido como Teorema de Ceva. Neste mesmo livro há citação do Teorema de Menelaus. O Teorema de Ceva trata de uma relação envolvendo um triângulo e as suas cevianas. A critério de curiosidade, na época de sua publicação, o resultado de Giovanni Ceva não teve grande repercussão e somente mais tarde o matemático francês Joseph Diaz Gergonne o redescobriu, e o autor, já falecido, ganhou os devidos méritos pela sua obra, como podemos ver em $[4]$.

Apesar de não serem tão abordados no currículo de Matemática do Ensino Básico e até mesmo nos cursos de nível de graduação em Matemática, os Teoremas de Menelaus e de Ceva têm tido um destaque relevante nas Olimpíadas de Matemática. Em nível nacional, esses resultados são abordados, por exemplo, nos programas de treinamento olímpico em matemática, tais como o Programa de Iniciação Científica Jr. para a Obmep (PIC-Obmep) e o Programa Polos Olímpicos de Treinamento Intensivo (Poti). Internacionalmente também está presente em grande parte dos programas de treinamento para Olimpíadas de Matemática como podemos ver em [15].

No contexto nacional, diversos trabalhos abordando questões olímpicas de Matemática têm sido desenvolvidos no decorrer dos últimos anos, promovendo o interesse por tais competições. Por trazerem novas abordagens para temáticas antigas ou até mesmo inovações de técnicas de soluções, esses trabalhos são fortes ferramentas matemáticas e imprescindíveis para aqueles que se desafiam em Olimpíadas de Matemática. Destacamos a nacionalmente conhecida Revista Eureka!, atualmente com 41 edições, trazendo artigos com temáticas diversas e direcionadas especificamente para a OBM. Menos conhecida, mas com um conteúdo totalmente direcionado para Olimpíadas de Matemática em geral, há o jornal É Matemática, Oxente!, atualmente com 18 edições, desenvolvidos por um grupo de professores da UFRPE. Na revista Professor de Matemática On-line (PMO) há dois artigos que trazem temáticas semelhantes à que abordaremos neste trabalho. Em [6] os autores solucionam três problemas da IMO que envolvem o tema bases numéricas, com organização de ideias e estratégias de suporte matemático para que seja possível trabalhar essas questões. Por outro lado, com um cunho mais focado nas técnicas, [2] faz uma discussão sobre alguns tópicos da Álgebra e da Teoria dos Números, direcionando a implementação da teoria para a resolução de questões olímpicas. Por fim, não em contextos olímpicos, mas diretamente relacionados com o conteúdo deste trabalho, podemos citar [1,3,4,14] com uma vasta teoria sobre os Teoremas de Ceva e de Menelaus.

Inspirados nos trabalhos acima citados, nosso objetivo principal é mostrar a utilidade dos Teoremas de Menelaus e de Ceva na resolução de questões olímpicas de Matemática, abordando também entre alguns outros conteúdos de Geometria que são acessíveis desde o Ensino Básico, o que pode estimular professores e alunos no âmbito das competições de Matemática. Além disso, esses resultados têm como consequências outros resultados intimamente ligados com o contexto olímpico como os Teoremas de Desargues, de Pascal, de Pappus, e até mesmo a forma trigonométrica do Teorema de Ceva, que podem ser encontrados em [1] e [3]. Como curiosidade final, resolveremos uma questão olímpica que ilustra a ligação existente entre os dois resultados.

Este trabalho está dividido da seguinte maneira: na Seção 2 discutimos o Teorema de Menelaus e na Seção 3 o Teorema de Ceva. Na Seção 4 discutimos a relação existente entre os dois resultados 
e, por fim, na Seção 5 trazemos uma série de problemas complementares.

No decorrer deste trabalho, dados quaisquer dois pontos $\mathrm{A}$ e B, denotaremos por $\mathrm{AB}$ o segmento de reta que une os pontos $\mathrm{A}$ e $\mathrm{B}$; por $\overline{\mathrm{AB}}$, a medida do segmento de reta $\mathrm{AB}$, e por $\overleftrightarrow{\mathrm{AB}}$ a reta que passa por $\mathrm{A}$ e $\mathrm{B}$. Denotaremos também por $\triangle \mathrm{ABC}$ o triângulo definido por três pontos não colineares $\mathrm{A}, \mathrm{B}$ e $\mathrm{C}$; por $\angle \mathrm{ABC}$ o ângulo definido por $\mathrm{AB}$ e $\mathrm{BC}$. Ademais, a área de $\triangle \mathrm{ABC}$ será denotada por $[\mathrm{ABC}]$, e quando $\triangle \mathrm{ABC}$ for semelhante a $\triangle \mathrm{XYZ}$, escreveremos $\triangle \mathrm{ABC} \sim \Delta \mathrm{XYZ}$. Todas as figuras ilustradas aqui foram desenvolvidas pelos autores no software livre GeoGebra.

\section{O Teorema de Menelaus}

A demonstração do Teorema de Menelaus será feita utilizando o conceito de semelhança de triângulos. É importante destacar que há outras maneiras de demonstrar esse resultado utilizando diversas ferramentas como o Teorema de Tales, medidas de áreas, Trigonometria (lei dos senos), Geometria Analítica, entre outras (ver [4, 14]). Sendo assim, observamos que esse tema é bastante acessível ainda no Ensino Básico.

Teorema 1 (Menelaus). Dado um triângulo $\triangle \mathrm{ABC}$ qualquer, sejam $\mathrm{X}$ um ponto no prolongamento do lado $\mathrm{BC}, \mathrm{Y}$ e $\mathrm{Z}$ pontos pertencentes aos lados $\mathrm{AC}$ e $\mathrm{AB}$, respectivamente (ver Figura 1).

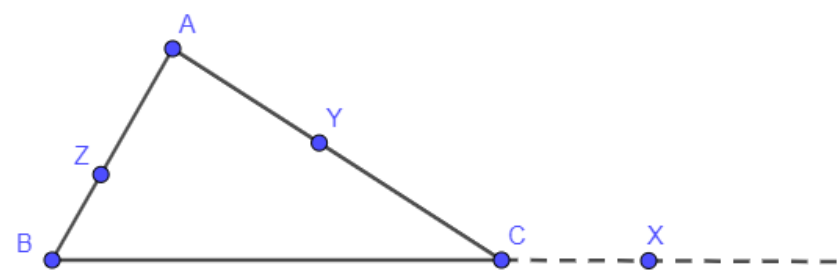

Figura 1: Figura suporte para o enunciado do Teorema 1. (Fonte: Autores.)

Os pontos $\mathrm{X}, \mathrm{Y}$ e $\mathrm{Z}$ são colineares, se e somente se,

$$
\frac{\overline{\mathrm{AZ}}}{\overline{\mathrm{BZ}}} \cdot \frac{\overline{\mathrm{BX}}}{\overline{\mathrm{CX}}} \cdot \frac{\overline{\mathrm{CY}}}{\overline{\mathrm{AY}}}=1 .
$$

Nessa situação a Figura 1 assume o seguinte formato

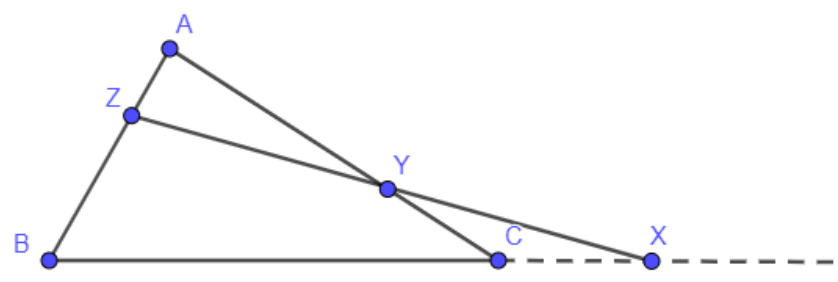

Figura 2: Situação em que os pontos X, Y e Z são colineares. (Fonte: Autores.)

Demonstração. $(\Rightarrow)$ Suponha que os pontos X, Y e Z são colineares. Construa um ponto $\mathrm{G} \in \overleftrightarrow{\mathrm{XZ}}$ de modo que o segmento CG seja paralelo ao lado AB, como ilustra a Figura 3. 


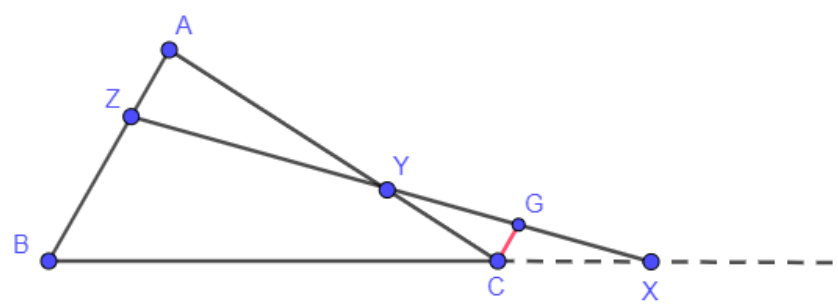

Figura 3: Figura suporte para demonstração do Teorema 1. (Fonte: Autores.)

Além disso, ainda pela Figura 3, observamos através do caso de semelhança Ângulo-Ângulo (A.A.) que $\Delta \mathrm{CGY} \sim \Delta \mathrm{AZY}$, e portanto, $\frac{\overline{\mathrm{AZ}}}{\overline{\mathrm{CG}}}=\frac{\overline{\mathrm{AY}}}{\overline{\mathrm{CY}}}$. Para forçar o aparecimento da identidade que desejamos provar, multiplicaremos a identidade anterior por $\frac{\overline{\mathrm{CG}}}{\overline{\mathrm{BZ}}}$ e ficaremos com:

$$
\frac{\overline{\mathrm{AZ}}}{\overline{\mathrm{CG}}} \cdot \frac{\overline{\mathrm{CG}}}{\overline{\mathrm{BZ}}}=\frac{\overline{\mathrm{AY}}}{\overline{\mathrm{CY}}} \cdot \frac{\overline{\mathrm{CG}}}{\overline{\mathrm{BZ}}} .
$$

$\mathrm{Na}$ identidade acima, simplificando o termo $\overline{\mathrm{CG}}$ do lado esquerdo da igualdade e, em seguida, passando todos os termos do lado direito para o lado esquerdo da igualdade, ficamos com

$$
\frac{\overline{\mathrm{AZ}}}{\overline{\mathrm{BZ}}} \cdot \frac{\overline{\mathrm{BZ}}}{\overline{\mathrm{CG}}} \cdot \frac{\overline{\mathrm{CY}}}{\overline{\mathrm{AY}}}=1 .
$$

Ainda pelo caso Ângulo-Ângulo (A.A.), temos que $\Delta \mathrm{CGX} \sim \Delta \mathrm{BZX}$, e portanto, $\frac{\overline{\mathrm{BZ}}}{\overline{\mathrm{CG}}}=\frac{\overline{\mathrm{BX}}}{\overline{\mathrm{CX}}}$. Fazendo essa substituição na equação anterior, obtemos a identidade desejada, isto é,

$$
\frac{\overline{\mathrm{AZ}}}{\overline{\mathrm{BZ}}} \cdot \frac{\overline{\mathrm{BX}}}{\overline{\mathrm{CX}}} \cdot \frac{\overline{\mathrm{CY}}}{\overline{\mathrm{AY}}}=1 .
$$

$(\Leftarrow)$ Assumindo agora que $\frac{\overline{\mathrm{AZ}}}{\overline{\mathrm{BZ}}} \cdot \frac{\overline{\mathrm{BX}}}{\overline{\mathrm{CX}}} \cdot \frac{\overline{\mathrm{CY}}}{\overline{\mathrm{AY}}}=1$, provaremos que os pontos X, Y Z são colineares. Seja $\mathrm{Z}^{\prime}$ um ponto sobre o lado $\mathrm{AB}$ tal que $\mathrm{X}, \mathrm{Y}$ e $\mathrm{Z}^{\prime}$ são colineares como mostra a Figura 4. Provaremos que $\mathrm{Z}=\mathrm{Z}^{\prime}$.

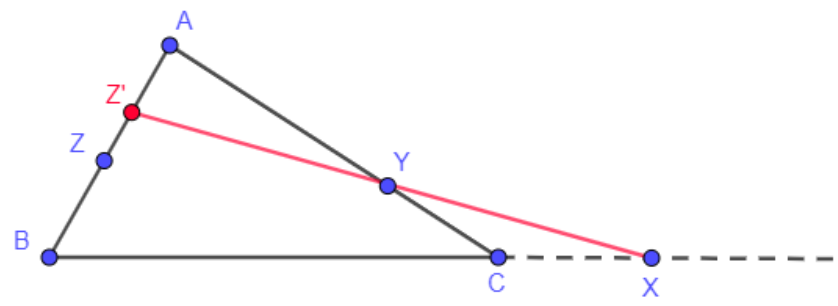

Figura 4: Figura suporte da demonstração do Teorema 1. (Fonte: Autores.) 
Pela primeira parte da demonstração (que já demonstramos ser verdade), como os pontos X, Y e $\mathrm{Z}^{\prime}$ são colineares, vale a equação $\frac{\overline{\mathrm{AZ}}}{\overline{\mathrm{BZ}^{\prime}}} \cdot \frac{\overline{\mathrm{BX}}}{\overline{\mathrm{CX}}} \cdot \frac{\overline{\mathrm{CY}}}{\overline{\mathrm{AY}}}=1$. Portanto,

$$
\frac{\overline{\mathrm{AZ}}}{\overline{\mathrm{BZ}}} \cdot \frac{\overline{\mathrm{BX}}}{\overline{\mathrm{CX}}} \cdot \frac{\overline{\mathrm{CY}}}{\overline{\mathrm{AY}}}=1=\frac{\overline{\mathrm{AZ}^{\prime}}}{\overline{\mathrm{BZ}^{\prime}}} \cdot \frac{\overline{\mathrm{BX}}}{\overline{\mathrm{CX}}} \cdot \frac{\overline{\mathrm{CY}}}{\overline{\mathrm{AY}}},
$$

após a simplificação dos termos em comum, concluímos que

$$
\frac{\overline{\mathrm{AZ}}}{\overline{\mathrm{BZ}}}=\frac{\overline{\mathrm{AZ}^{\prime}}}{\overline{\mathrm{BZ}^{\prime}}}
$$

Como $\mathrm{Z}$ e $\mathrm{Z}^{\prime}$ são pontos pertencentes ao lado $\mathrm{AB}$, temos que a única possibilidade da equação (1) ocorrer é $\mathrm{Z}=\mathrm{Z}^{\prime}$, como queríamos demonstrar.

Observação. Note que, no caso em que X, Y e Z pertencem todos aos prologamentos dos lados do triângulo $\triangle \mathrm{ABC}$, o teorema ainda é válido.

As questões mais comuns para aplicação de Menelaus são do tipo em que há uma série de construções geométricas e no fim pede-se para mostrar que determinados pontos estão sob uma mesma reta ou segmento de reta, ou seja, são ligados diretamente com problemas envolvendo colinearidade de pontos.

Exemplo 1 (IMO/1982). As diagonais AC e CE de um hexágono regular ABCDEF são divididas internamente pelos pontos $\mathrm{M}$ e $\mathrm{N}$, respectivamente, na razão

$$
\frac{\overline{\mathrm{AM}}}{\overline{\mathrm{AC}}}=\frac{\overline{\mathrm{CN}}}{\overline{\mathrm{CE}}}=\mathrm{r} \text {. }
$$

Encontre o valor r uma vez que B, M e N são pontos colineares.

Solução. Construa a diagonal BE e seja $\mathrm{P}$ o ponto de interseção dessa diagonal com a diagonal AC, conforme a construção abaixo:

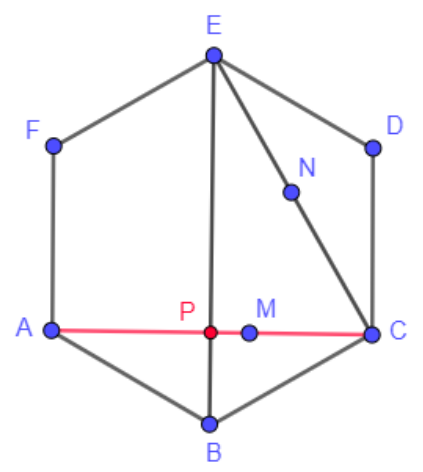

Figura 5: Figura do exemplo 1. (Fonte: Autores.) 
Utilizando agora o Teorema de Menalaus considerando o triângulo $\triangle \mathrm{CEP}$ e os pontos $\mathrm{B}, \mathrm{M}$ e $\mathrm{N}$ (que por hipótese são colineares), obtemos que

$$
\frac{\overline{\mathrm{CM}}}{\overline{\mathrm{MP}}} \cdot \frac{\overline{\mathrm{PB}}}{\overline{\mathrm{BE}}} \cdot \frac{\overline{\mathrm{EN}}}{\overline{\mathrm{NC}}}=1 \text {. }
$$

Vamos agora descobrir os valores dessas três razões. Utilizando o princípio aditivo, perceba que podemos reescrever os tamanhos dos segmentos CM e MP em termos dos segmentos AM e AC da seguinte forma:

$$
\overline{\mathrm{CM}}=\overline{\mathrm{AC}}-\overline{\mathrm{AM}}
$$

e

$$
\overline{\mathrm{MP}}=\overline{\mathrm{AM}}-\overline{\mathrm{AP}}=\overline{\mathrm{AM}}-\frac{\overline{\mathrm{AC}}}{2} .
$$

Assim, reescrevendo a expressão $\frac{\overline{\mathrm{CM}}}{\overline{\mathrm{MP}}}$ em termos de (3) e (4), em seguida, forçando o aparecimento de $\frac{\overline{\mathrm{AM}}}{\overline{\mathrm{AC}}}=\mathrm{r}$, ficamos com

$$
\frac{\overline{\mathrm{CM}}}{\overline{\mathrm{MP}}}=\frac{\overline{\mathrm{AC}}-\overline{\mathrm{AM}}}{\overline{\mathrm{AM}}-\frac{\overline{\mathrm{AC}}}{2}}=\frac{\frac{\overline{\mathrm{AC}}-\overline{\mathrm{AM}}}{\overline{\mathrm{AC}}}}{\frac{\overline{\mathrm{AM}}-\frac{\overline{\mathrm{AC}}}{2}}{\overline{\mathrm{AC}}}}=\frac{\frac{\overline{\mathrm{AC}}}{\overline{\mathrm{AM}}}-\frac{\overline{\mathrm{AM}}}{\overline{\mathrm{AC}}}}{\frac{\overline{\mathrm{AC}}}{\overline{\mathrm{AC}}}}=\frac{1-\mathrm{r}}{\mathrm{2} \overline{\mathrm{AC}}}=\frac{1}{2}=\frac{2 \mathrm{r}}{2 \mathrm{r}-1}
$$

Para a segunda razão, observaremos a diagonal BE. Uma primeira observação é que ela mede o dobro da medida do lado do hexágono. Note também que essa diagonal divide o ângulo interno $\angle \mathrm{ABC}$ em duas medidas congruentes, e assim o ângulo $\mathrm{ABP}=60^{\circ}$. Utilizando trigonometria no triângulo $\triangle \mathrm{ABP}$, concluímos que

$$
\overline{\mathrm{PB}}=\overline{\mathrm{AB}} \cos \left(60^{\circ}\right)=\frac{\overline{\mathrm{AB}}}{2}=\frac{\frac{\overline{\mathrm{BE}}}{2}}{2}=\frac{\overline{\mathrm{BE}}}{4} .
$$

Portanto,

$$
\overline{\overline{\mathrm{BE}}}=\frac{1}{4}
$$

Por fim, novamente pelo princípio aditivo, perceba que $\overline{\mathrm{EN}}=\overline{\mathrm{CE}}-\overline{\mathrm{CN}}$. Dividindo essa igualdade por $\overline{\mathrm{CN}}$ (para forçar aparecer a razão que queremos) e usando que $\frac{\overline{\mathrm{CN}}}{\overline{\mathrm{CE}}}=\mathrm{r}$, ficamos com

$$
\frac{\overline{\mathrm{EN}}}{\overline{\mathrm{CN}}}=\frac{\overline{\mathrm{CE}}}{\overline{\mathrm{CN}}}-\frac{\overline{\mathrm{CN}}}{\overline{\mathrm{CN}}}=\frac{1}{\mathrm{r}}-1=\frac{1-\mathrm{r}}{\mathrm{r}} .
$$

Substituindo na identidade (2) as informações obtidas em (5), (6) e (7), obtemos

$$
\frac{2-2 \mathrm{r}}{2 \mathrm{r}-1} \cdot \frac{1}{4} \cdot \frac{1-\mathrm{r}}{\mathrm{r}}=1
$$


Segue desta igualdade que $(1-r)^{2}=4 r^{2}-2 r$, que é uma equação quadrática cujas soluções são $r= \pm \sqrt{\frac{1}{3}}$. Sabendo que $r$ é um número maior que zero, pois é a razão de duas medidas, temos que a única solução é $r=\sqrt{\frac{1}{3}}$.

Veremos no exemplo a seguir que nem sempre o Teorema de Menelaus dá-nos a solução mais simples e direta para um determinado problema. Isso é um alerta. Mesmo tendo essa ferramenta em mãos, é importante não esquecer que se pode usar quaisquer outros artifícios quando for resolver uma questão olímpica.

Exemplo 2 (POTI - Geometria - Nível 2). O lado AB de um quadrado é prolongado no sentido de $\mathrm{A}$ para $\mathrm{B}$ até o ponto $\mathrm{P}$ tal que $\overline{\mathrm{BP}}=2 \overline{\mathrm{AB}}$. Sendo $\mathrm{M}$ o ponto médio de $\mathrm{CD}$, desenhe $\mathrm{PM}$ intersectando AC em um ponto Q. Sabendo que PQ corta BC em um ponto R, calcule $\overline{\mathrm{CR}} / \overline{\mathrm{RB}}$.

Solução. Neste exemplo iremos construir duas soluções, uma utilizando o Teorema de Menelaus e outra não.

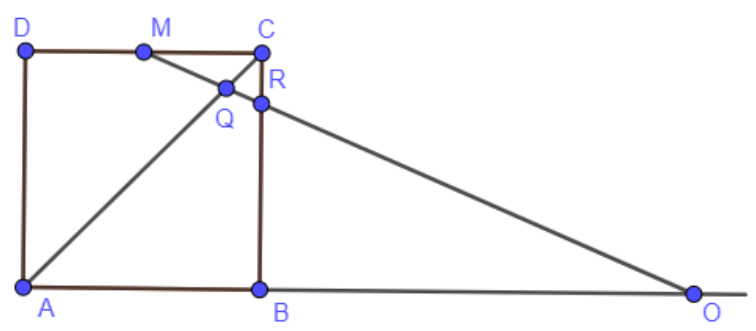

Figura 6: Figura de suporte para o exemplo 2. (Fonte: Autores.)

Denote por x o comprimento do lado do quadrado em questão. Para a solução com o Teorema de Menelaus, primeiramente observe que pelo caso Ângulo-Ângulo (A.A.) os triângulos $\Delta$ APQ e $\Delta \mathrm{CQM}$ são semelhantes. Isso nos garante que

$$
\frac{\overline{\mathrm{CQ}}}{\overline{\mathrm{AQ}}}=\frac{\overline{\mathrm{CM}}}{\overline{\mathrm{AP}}}=\frac{\bar{x}}{3 \mathrm{x}}=\frac{1}{6} \text {. }
$$

Como os pontos P, R e Q são colineares, podemos aplicar o Teorema de Menelaus ao triângulo $\triangle \mathrm{ABC}$ com o segmento $\mathrm{PQ}$ e concluir que

$$
\frac{\overline{\mathrm{CQ}}}{\overline{\mathrm{AQ}}} \cdot \frac{\overline{\mathrm{AP}}}{\overline{\mathrm{BP}}} \cdot \frac{\overline{\mathrm{BR}}}{\overline{\mathrm{CR}}}=1 .
$$

Além disso, como $\overline{\mathrm{BP}}=2 \overline{\mathrm{AB}}$, então

$$
\frac{\overline{\mathrm{AP}}}{\overline{\mathrm{BP}}}=\frac{3}{2} .
$$

Utilizando as informações que temos, podemos concluir que

$$
\frac{1}{6} \cdot \frac{3}{2} \cdot \frac{\overline{\mathrm{BR}}}{\overline{\mathrm{CR}}}=1
$$


e assim,

$$
\frac{\overline{\mathrm{CR}}}{\overline{\mathrm{BR}}}=\frac{1}{4}
$$

Uma outra solução que é mais simples do que a apresentada acima, é observar que, pelo caso Ângulo-Ângulo (A.A.) os triângulos $\triangle \mathrm{CRM}$ e $\triangle \mathrm{BPR}$ são semelhantes. Daí temos que

$$
\frac{\overline{\mathrm{CR}}}{\overline{\mathrm{RB}}}=\frac{\overline{\mathrm{CM}}}{\overline{\mathrm{BP}}}=\frac{\overline{\mathrm{x}}}{2 \mathrm{x}}=\frac{1}{4} \text {. }
$$

Exemplo 3 (Retirado de [7]). Em um paralelogramo ABCD com ângulo $\angle \mathrm{BA} D<90^{\circ}$, o círculo com diâmetro $\mathrm{AC}$ intersecta as retas $\overleftrightarrow{\mathrm{CB}}$ e $\overleftrightarrow{\mathrm{CD}}$ nos pontos $\mathrm{E}$ e F respectivamente, e a reta tangente a esse círculo no ponto $\mathrm{A}$ intersecta $\overleftrightarrow{\mathrm{BD}}$ no ponto $\mathrm{P}$. Prove que $\mathrm{P}, \mathrm{F}$ e E são colineares.

Solução. Sem perda de generalidade, suponha que B, D e P estão nessa ordem ao longo de BD.

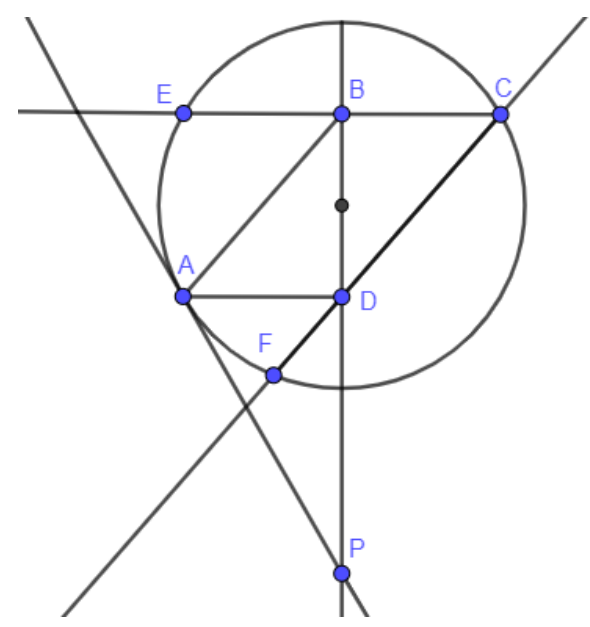

Figura 7: Figura de suporte para o exemplo 3. (Fonte: Autores.)

Sejam G e H a segunda interseção de AD e AB, respectivamente, com o círculo. Pelo Teorema de Menelaus com o triângulo $\Delta \mathrm{CBD}$ e a reta suporte $\stackrel{\mathrm{EF}}{\text { }}$, é suficiente mostrar que

$$
\frac{\overline{\mathrm{CE}}}{\overline{\mathrm{EB}}} \cdot \frac{\overline{\mathrm{BP}}}{\overline{\mathrm{PD}}} \cdot \frac{\overline{\mathrm{DF}}}{\overline{\overline{\mathrm{FC}}}}=1 \text {. }
$$

Primeiramente, usando lei dos senos, note que:

$$
\frac{\overline{\mathrm{BP}}}{\overline{\mathrm{AB}}} \cdot \frac{\overline{\mathrm{AD}}}{\overline{\mathrm{DP}}}=\frac{\operatorname{sen}(\angle \mathrm{BA} \mathrm{P})}{\operatorname{sen}(\angle \mathrm{AP} \mathrm{P})} \cdot \frac{\operatorname{sen}(\angle \mathrm{APD})}{\operatorname{sen}(\angle \mathrm{D} \hat{\mathrm{AP}})}=\frac{\operatorname{sen}(\angle \mathrm{BA} \mathrm{P})}{\operatorname{sen}(\angle \mathrm{DA} \mathrm{AP})}
$$

como AP é tangente ao circulo, perceba que $\angle \mathrm{BAP}=\angle \mathrm{HAP}=\pi-\angle \mathrm{HAC}=\pi-\angle \mathrm{FAC}$. De maneira análoga, $\angle \mathrm{DA} \mathrm{P}=\angle \mathrm{G} \hat{\mathrm{CA}}=\angle \mathrm{EAC}$ e assim concluímos que 


$$
\frac{\overline{\mathrm{BP}}}{\overline{\mathrm{AB}}} \cdot \frac{\overline{\mathrm{AD}}}{\overline{\mathrm{DP}}}=\frac{\operatorname{sen}(\angle \mathrm{FA} C)}{\operatorname{sen}(\angle \mathrm{EACC})}=\frac{\overline{\mathrm{FA}}}{\overline{\mathrm{EC}}} .
$$

Finalmente, temos que

$$
\frac{\overline{\mathrm{DF}}}{\overline{\mathrm{BE}}}=\frac{\overline{\mathrm{DA}}}{\overline{\mathrm{AB}}},
$$

pois os triângulos $\triangle \mathrm{AFD}$ e $\triangle \mathrm{AEB}$ possuem os mesmos ângulos em $\mathrm{B}$ e $\mathrm{D}$, sendo assim são semelhantes.

Finalizaremos esta seção com a utilização do Teorema de Menelaus para demonstrar outro resultado utilizado em Olimpíadas de Matemática que é o Teorema de Pascal.

Teorema 2 (Pascal). Seja ABCDEF um hexágono inscrito em uma circunferência. Então, os pontos de interseções $\overleftrightarrow{\mathrm{AB}} \cap \overleftrightarrow{\mathrm{DE}}, \overleftrightarrow{\mathrm{BC}} \cap \overleftrightarrow{\mathrm{EF}}$ e $\overleftrightarrow{\mathrm{CD}} \cap \overleftrightarrow{\mathrm{FA}}$ são colineares.

Demonstração. Para esta demonstração iremos denotar por X, Y, Z, respectivamente as interseções $\overleftrightarrow{\mathrm{AB}} \cap \overleftrightarrow{\mathrm{DE}}, \overleftrightarrow{\mathrm{BC}} \cap \overleftrightarrow{\mathrm{EF}}$ e $\overleftrightarrow{\mathrm{CD}} \cap \overleftrightarrow{\mathrm{FA}}$

Sejam M a interseção de $\overleftrightarrow{\mathrm{CD}}$ com $\overleftrightarrow{\mathrm{EF}}, \mathrm{N}$ a interseção de $\overleftrightarrow{\mathrm{AB}}$ com $\overleftrightarrow{\mathrm{CD}}$ e $\mathrm{O}$ a interseção de $\overleftrightarrow{\mathrm{AB}}$ com $\overleftrightarrow{\mathrm{EF}}$. Como mostra a Figura 8

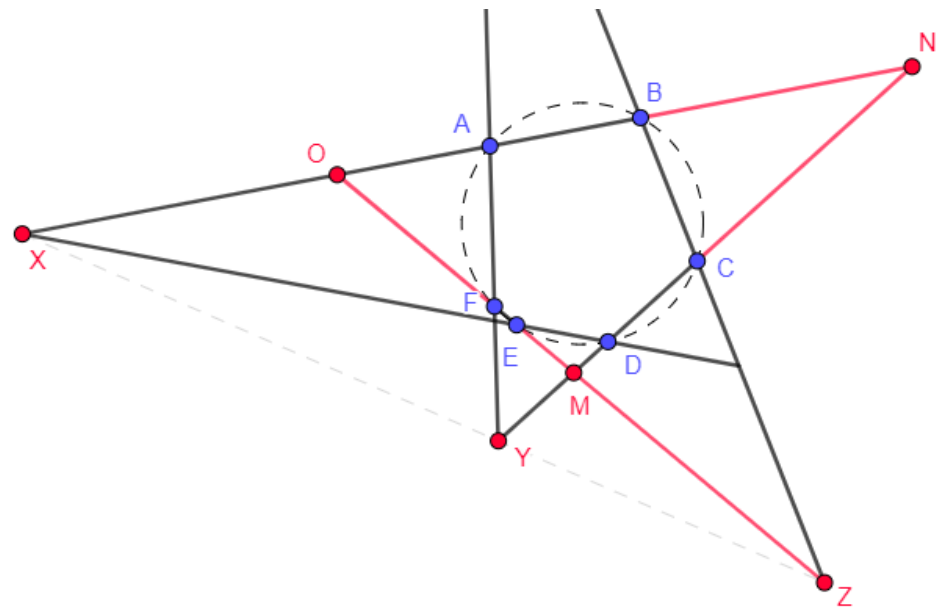

Figura 8: Figura suporte para a demonstração do Teorema de Pascal. (Fonte: Autores.)

Logo, aplicando o Teorema de Menelaus no triângulo $\Delta \mathrm{MNO}$ e nos pontos colineares X, D, E, temos:

$$
\frac{\overline{\mathrm{NX}}}{\overline{\mathrm{OX}}} \cdot \frac{\overline{\mathrm{OE}}}{\overline{\mathrm{ME}}} \cdot \frac{\overline{\mathrm{MD}}}{\overline{\mathrm{ND}}}=1,
$$

de maneira análoga, podemos fazer para o mesmo triângulo $\Delta \mathrm{MNO}$ e nos pontos colineares $\mathrm{Y}, \mathrm{C}, \mathrm{B}$, e teremos:

$$
\frac{\overline{\mathrm{YM}}}{\overline{\mathrm{OY}}} \cdot \frac{\overline{\mathrm{OB}}}{\overline{\mathrm{NB}}} \cdot \frac{\overline{\mathrm{NC}}}{\overline{\mathrm{MC}}}=1 \text {, }
$$


e ainda para o triângulo $\triangle \mathrm{MNO}$ nos pontos colineares $\mathrm{Z}, \mathrm{F}, \mathrm{A}$

$$
\frac{\overline{\mathrm{NZ}}}{\overline{\mathrm{MZ}}} \cdot \frac{\overline{\mathrm{MF}}}{\overline{\mathrm{OF}}} \cdot \frac{\overline{\mathrm{OA}}}{\overline{\mathrm{NA}}}=1 \text {. }
$$

Unindo as equações (8), (9) e (10), teremos

$$
\frac{\overline{\mathrm{NX}}}{\overline{\mathrm{OX}}} \cdot \frac{\overline{\mathrm{OE}}}{\overline{\mathrm{ME}}} \cdot \frac{\overline{\mathrm{MD}}}{\overline{\mathrm{ND}}} \cdot \frac{\overline{\mathrm{OY}}}{\overline{\mathrm{YM}}} \cdot \frac{\overline{\mathrm{NB}}}{\overline{\mathrm{OB}}} \cdot \frac{\overline{\mathrm{MC}}}{\overline{\mathrm{NC}}} \cdot \frac{\overline{\mathrm{MZ}}}{\overline{\mathrm{NZ}}} \cdot \frac{\overline{\mathrm{OF}}}{\overline{\mathrm{MF}}} \cdot \frac{\overline{\mathrm{NA}}}{\overline{\mathrm{OA}}}=1 .
$$

Reorganizando, teremos,

$$
\left(\frac{\overline{\mathrm{NX}}}{\overline{\mathrm{OX}}} \cdot \frac{\overline{\mathrm{OY}}}{\overline{\mathrm{YM}}} \cdot \frac{\overline{\mathrm{MZ}}}{\overline{\mathrm{NZ}}}\right) \cdot\left(\frac{\overline{\mathrm{OE}}}{\overline{\mathrm{ME}}} \cdot \frac{\overline{\mathrm{MD}}}{\overline{\mathrm{ND}}} \cdot \frac{\overline{\mathrm{NB}}}{\overline{\mathrm{OB}}} \cdot \frac{\overline{\mathrm{MC}}}{\overline{\mathrm{NC}}} \cdot \frac{\overline{\mathrm{OF}}}{\overline{\mathrm{MF}}} \cdot \frac{\overline{\mathrm{NA}}}{\overline{\mathrm{OA}}}\right)=1
$$

Usando a condição da potência do ponto, teremos que o lado direito de (12) pode ser calculado da seguinte maneira

$$
\frac{\overline{\mathrm{OE}}}{\overline{\mathrm{ME}}} \cdot \frac{\overline{\mathrm{MD}}}{\overline{\mathrm{ND}}} \cdot \frac{\overline{\mathrm{NB}}}{\overline{\mathrm{OB}}} \cdot \frac{\overline{\mathrm{MC}}}{\overline{\mathrm{NC}}} \cdot \frac{\overline{\mathrm{OF}}}{\overline{\mathrm{MF}}} \cdot \frac{\overline{\mathrm{NA}}}{\overline{\mathrm{OA}}}=\frac{\overline{\mathrm{OE}} \cdot \overline{\mathrm{OF}}}{\overline{\mathrm{OB}} \cdot \overline{\mathrm{OA}}} \cdot \frac{\overline{\mathrm{MD}} \cdot \overline{\mathrm{MC}}}{\overline{\mathrm{ME}} \cdot \overline{\mathrm{MF}}} \cdot \frac{\overline{\mathrm{NB}} \cdot \overline{\mathrm{NA}}}{\overline{\mathrm{ND}} \cdot \overline{\mathrm{NC}}}=1 .
$$

Logo, por (12) e (13),

$$
\frac{\overline{\mathrm{NX}}}{\overline{\mathrm{OX}}} \cdot \frac{\overline{\mathrm{OY}}}{\overline{\mathrm{YM}}} \cdot \frac{\overline{\mathrm{MZ}}}{\overline{\mathrm{NZ}}}=1 \text {. }
$$

E como os pontos $\mathrm{X}, \mathrm{Y}, \mathrm{Z}$ pertencem aos prolongamentos dos lados do triângulo $\Delta \mathrm{MNO}$, podemos afirmar pelo Teorema de Menelaus que X, Y,Z são colineares, demonstrando assim o Teorema de Pascal.

Observação 1. No Teorema acima o hexágono não precisa ser convexo, ou seja, também pode ser degenerado.

\section{O Teorema de Ceva}

Vamos, nessa seção, enunciar e demonstrar o Teorema de Ceva. Comentaremos também sobre o Ponto de Gergonne (o mesmo da introdução deste trabalho). Começaremos definindo o conceito de ceviana.

Definição 1. Uma ceviana de um triângulo é um segmento de reta que liga um dos vértices a um ponto do lado oposto a esse vértice ou ao prologamento desse lado.

Portanto, se X, Y e Z são pontos nos lados BC, AC e AB ou nos seus prolongamentos, respectivamente, de um triângulo $\triangle \mathrm{ABC}$, os segmentos $\mathrm{AX}, \mathrm{BY}$ e $\mathrm{CZ}$ são denominados de cevianas, como mostra a Figura 9 

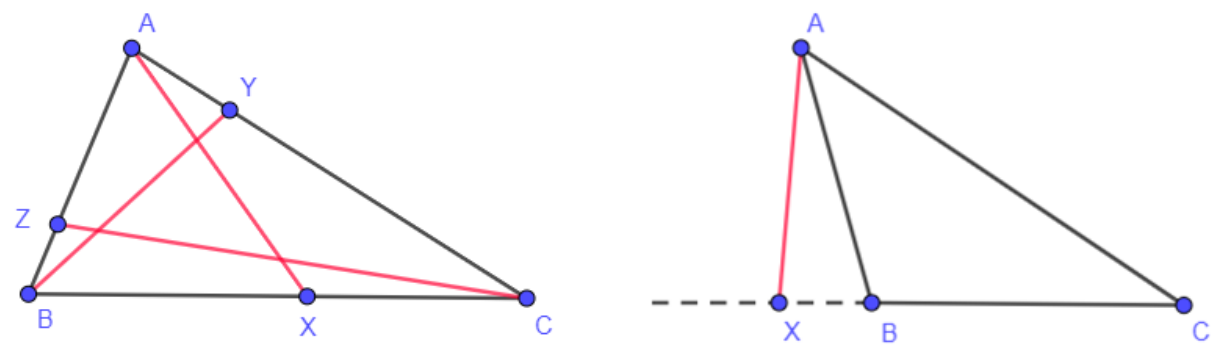

Figura 9: Figura de representação de cevianas. (Fonte: Autores.)

Agora, utilizando o Teorema de Menelaus, demonstraremos o Teorema de Ceva.

Teorema 3 (Ceva). Dado um triângulo $\triangle \mathrm{ABC}$ qualquer, considere as cevianas $\mathrm{AX}$, BY e CY, conforme a Figura 10. As cevianas são concorrentes, se e somente se,

$$
\frac{\overline{\mathrm{AZ}}}{\overline{\mathrm{BZ}}} \cdot \frac{\overline{\mathrm{BX}}}{\overline{\mathrm{CX}}} \cdot \frac{\overline{\mathrm{CY}}}{\overline{\mathrm{AY}}}=1 \text {. }
$$

Como mostra a figura abaixo

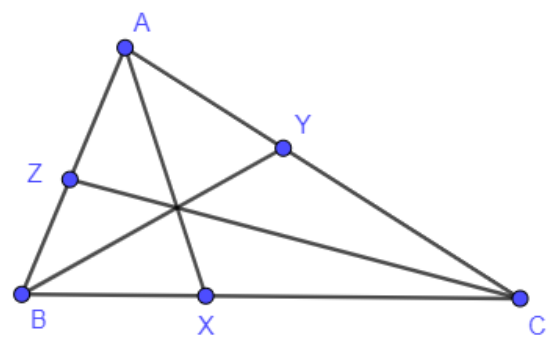

Figura 10: Teorema de Ceva. (Fonte: Autores.)

Demonstração. Como queremos demonstrar uma equivalência, primeiramente iremos supor que as cevianas $\overline{\mathrm{AX}}, \overline{\mathrm{BY}}$ e $\overline{\mathrm{CZ}}$ são concorrentes no ponto $\mathrm{P}$, e a partir disso provaremos que $\frac{\overline{\mathrm{AZ}}}{\overline{\mathrm{BZ}}} \cdot \overline{\overline{\mathrm{BX}}} \cdot \frac{\overline{\mathrm{CY}}}{\overline{\mathrm{AY}}}=1$. Da nossa hipótese podemos construir os triângulos conforme a figura abaixo
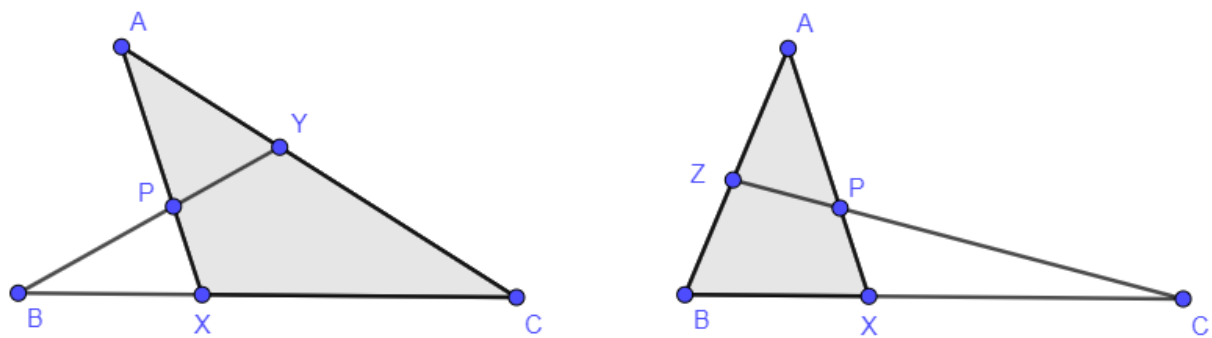

Figura 11: Triângulos $\triangle \mathrm{AXC}$ e $\triangle \mathrm{BAX}$. (Fonte: Autores.) 
Aplicando o Teorema de Menelaus, respectivamente, para os triângulos $\triangle \mathrm{AXC}$ com o segmento $\mathrm{BY}$ e $\triangle \mathrm{BAX}$ com o segmento $\mathrm{CZ}$, obtemos

$$
\begin{aligned}
& \frac{\overline{\mathrm{PA}}}{\overline{\mathrm{PX}}} \cdot \frac{\overline{\mathrm{BX}}}{\overline{\mathrm{BC}}} \cdot \frac{\overline{\mathrm{CY}}}{\overline{\mathrm{CA}}}=1, \\
& \frac{\overline{\mathrm{ZA}}}{\overline{\mathrm{ZB}}} \cdot \frac{\overline{\mathrm{BC}}}{\overline{\mathrm{CX}}} \cdot \frac{\overline{\mathrm{PX}}}{\overline{\mathrm{PA}}}=1 .
\end{aligned}
$$

Multiplicando (15) e (16), e simplificando os termos em comum, obtemos:

$$
\frac{\overline{\mathrm{AZ}}}{\overline{\mathrm{BZ}}} \cdot \frac{\overline{\mathrm{BX}}}{\overline{\mathrm{CX}}} \cdot \frac{\overline{\mathrm{CY}}}{\overline{\mathrm{AY}}}=1,
$$

o que prova uma das implicações. Resta mostrar a recíproca do teorema, ou seja, mostrar que se vale (17) então as cevianas são concorrentes em um único ponto. No entanto, essa parte da demonstração é análoga ao que fizemos para a volta do Teorema de Menelaus. Sendo assim, iremos omiti-la.

Observe que como o Teorema de Menelaus foi usado na demonstração do Teorema de Ceva, então podemos usar os mesmos métodos da demonstração que usamos no Teorema Menelaus para demonstrar o Teorema de Ceva.

As questões a serem exploradas a seguir decorrem do Teorema 3 e estão ligadas à concorrência entre segmentos e/ou retas. Nosso primeiro exemplo será uma curiosidade bastante interessante sobre este resultado.

Exemplo 4 (Autores). Apressado para chegar na prova de desenho geométrico, Victor Avelar percebeu que havia esquecido sua régua e seu compasso, e seu único instrumento era um par de esquadros que até serviria de régua, mas não possuía marcação. Na primeira questão de sua prova eram dados dois pontos A e B e pedia-se para encontrar o ponto médio do segmento AB. Vendo o desespero do seu amigo, Ferreira Yukio entregou-lhe secretamente um bilhete escrito "lembre do Teorema de Ceva". Em seguida, Victor Avelar, apenas com seu lápis e o par de esquadros conseguiu obter a solução para esse problema. Como ele fez isso?

Solução. Victor Avelar fez uma série de construções para aplicar o Teorema de Menelaus. Primeiramente, usando um de seus esquadros como régua, ele criou uma reta que passava pelos pontos A e B e, em seguida, usando o par de esquadros ele construiu uma reta paralela à primeira reta, conforme a figura abaixo:

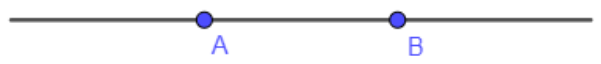

Figura 12: Figura da prova de Victor Avelar. (Fonte: Autores.)

Ele pegou um ponto $\mathrm{C}$ acima das duas retas, construiu um triângulo $\triangle \mathrm{ABC}$ e, em seguida, lembrando do Teorema de Ceva, traçou as cevianas BE e CF; passando pela interseção dessas cevianas, construiu a terceira ceviana CM, como mostra a Figura 13 


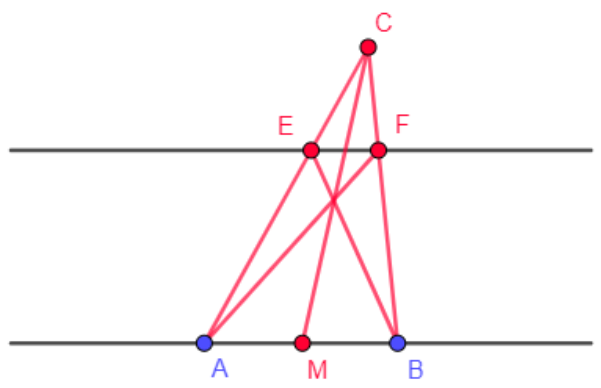

Figura 13: Figura da resposta de Victor Avelar. (Fonte: Autores.)

Daí, utilizando o Teorema de Ceva, Victor Avelar sabe que é verdadeira a seguinte identidade

$$
\frac{\overline{\mathrm{CE}}}{\overline{\mathrm{AE}}} \cdot \frac{\overline{\mathrm{AM}}}{\overline{\mathrm{BM}}} \cdot \frac{\overline{\mathrm{BF}}}{\overline{\mathrm{CF}}}=1 \text {. }
$$

Como a reta $\overleftrightarrow{\mathrm{AB}}$ é paralela à $\overleftrightarrow{\mathrm{EF}}$, usando o Teorema de Tales temos que

$$
\frac{\overline{\mathrm{CE}}}{\overline{\mathrm{AE}}} \cdot \frac{\overline{\mathrm{BF}}}{\overline{\mathrm{CF}}}=1 .
$$

Substituindo (19) em (18), obtém-se que

$$
\overline{\overline{\mathrm{BM}}}=1 \text {, }
$$

ou seja, $\overline{\mathrm{AM}}=\overline{\mathrm{BM}}$, o que prova que Victor Avelar conseguiu o ponto médio do segmento $\mathrm{AB}$.

Como aplicação do Teorema de Ceva, explicaremos em detalhes um fato que frequentemente utilizamos nas aulas de Desenho Geométrico, que é o fato de as três alturas de um triângulo intersectarem-se em um mesmo ponto (o ortocentro).

Exemplo 5 (Poti - Geometria - Nível 2). Prove que as três alturas de um triângulo são concorrentes em um único ponto.

Solução. Sejam o triângulo $\triangle \mathrm{ABC}$ e AX, BY e CZ suas alturas, conforme a figura abaixo:

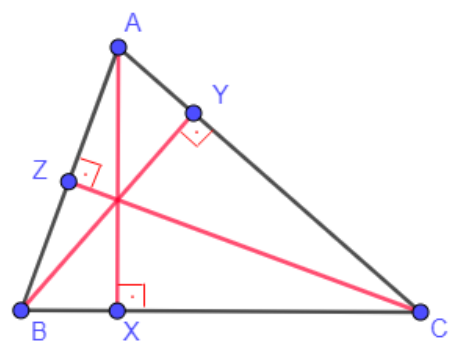

Figura 14: Alturas do triângulo $\triangle \mathrm{ABC}$. (Fonte: Autores.) 
Pelo caso Ângulo-Ângulo (A.A.) de semelhança, os triângulos $\triangle \mathrm{ACZ}$ e $\triangle \mathrm{ABY}$ são semelhantes, pois ambos possuem o ângulo no vértice A e um ângulo igual 90², assim, pela razão de semelhança, temos que

$$
\frac{\overline{\mathrm{AZ}}}{\overline{\mathrm{AY}}}=\frac{\overline{\mathrm{AC}}}{\overline{\overline{\mathrm{AB}}}} .
$$

Analogamente, temos as seguintes relações de semelhança: $\triangle \mathrm{ABX} \sim \triangle \mathrm{BCZ}$ e $\Delta \mathrm{BCY} \sim \Delta \mathrm{ACX}$, valendo assim as relações

$$
\frac{\overline{\mathrm{BX}}}{\overline{\mathrm{BZ}}}=\frac{\overline{\mathrm{AB}}}{\overline{\overline{\mathrm{BC}}}} \quad \text { e } \quad \frac{\overline{\mathrm{CY}}}{\overline{\mathrm{CX}}}=\frac{\overline{\mathrm{BC}}}{\overline{\mathrm{AC}}} .
$$

Multiplicando todas essas identidades no mesmo sentido vamos obter:

$$
\frac{\overline{\mathrm{AZ}}}{\overline{\mathrm{AY}}} \cdot \frac{\overline{\mathrm{BX}}}{\overline{\mathrm{BZ}}} \cdot \frac{\overline{\mathrm{CY}}}{\overline{\mathrm{CX}}}=\frac{\overline{\mathrm{AC}}}{\overline{\overline{\mathrm{AB}}}} \cdot \frac{\overline{\mathrm{AB}}}{\overline{\mathrm{BC}}} \cdot \frac{\overline{\mathrm{BC}}}{\overline{\mathrm{AC}}}=1 .
$$

Portanto, pelo Teorema de Ceva, as alturas são concorrentes em um único ponto.

Analogamente, pode-se mostrar que as bissetrizes e as medianas de um triângulo intersectam-se em um único ponto, mas isso ficará como um dos Problemas Propostos ao fim do trabalho. Falaremos agora sobre um tema olímpico decorrente do Teorema de Ceva.

Exemplo 6 (Ponto de Gergonne). Sejam D, E e F os pontos de contato da circunferência inscrita com os lados $\mathrm{BC}, \mathrm{CA}$ e $\mathrm{AB}$, respectivamente, do triângulo $\triangle \mathrm{ABC}$. Prove que $\mathrm{AD}, \mathrm{BE}$ e $\mathrm{CF}$ são concorrentes em um ponto (que se chama Ponto de Gergonne).

Solução. Observe que $\overline{\mathrm{BD}}=\overline{\mathrm{BF}}, \overline{\mathrm{AE}}=\overline{\mathrm{AF}}, \overline{\mathrm{CD}}=\overline{\mathrm{CE}}$. Daí podemos concluir que

$$
\frac{\overline{\mathrm{CE}}}{\overline{\mathrm{AE}}} \cdot \frac{\overline{\mathrm{AF}}}{\overline{\mathrm{BF}}} \cdot \frac{\overline{\mathrm{BD}}}{\overline{\mathrm{CD}}}=1 \text {. }
$$

Portanto, pelo Teorema de Ceva, temos que AD, BE e CF são concorrentes em um ponto (que se chama Ponto de Gergonne), como mostra a figura abaixo.

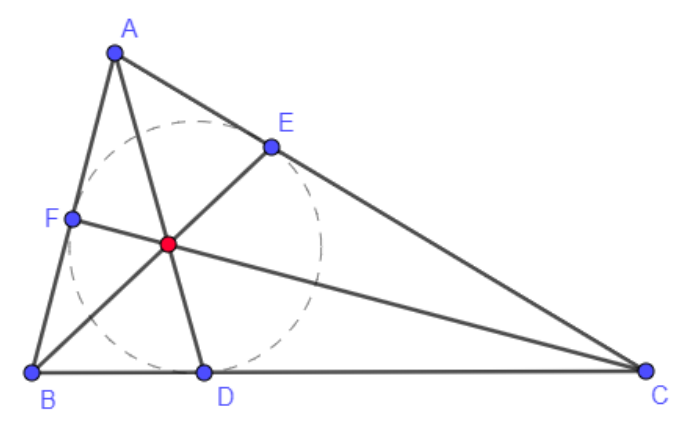

Figura 15: O ponto de Gergonne. (Fonte: Autores.)

Exemplo 7 (Bulgaria National Constest/1996). Os círculos $\mathrm{k}_{1}$ e $\mathrm{k}_{2}$ cujos centros são respectivamente os pontos $\mathrm{O}_{1}$ e $\mathrm{O}_{2}$, são tangentes externas em um ponto $\mathrm{C}$, enquanto que o círculo $\mathrm{k}$ com 
centro em $\mathrm{O}$ é tangente externa a $\mathrm{k}_{1}$ e $\mathrm{k}_{2}$. Seja $\mathrm{l}$ a reta tangente tanto a $\mathrm{k}_{1}$ e $\mathrm{k}_{2}$ passando por $\mathrm{C}$, e seja $\mathrm{AB}$ o diâmetro de $\mathrm{k}$ que é perpendicular a l. Assuma que $\mathrm{O}$ e A estão do mesmo lado de $\mathrm{l}$. Prove que as retas $\overleftrightarrow{\mathrm{AO}_{1}}, \overleftrightarrow{\mathrm{AO}_{2}}$ e 1 duas a duas possuem um ponto em comum.

Demonstração. A seguir construímos uma figura para melhor visualização do problema em questão:

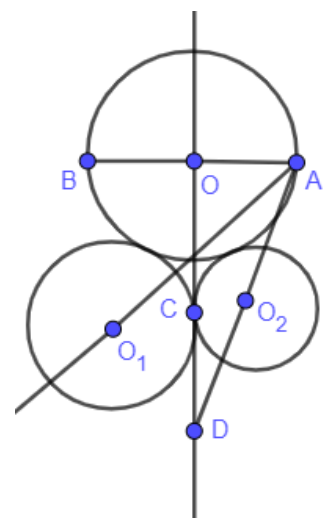

Figura 16: Figura para resolução do exemplo 7. (Fonte: Autores.)

Tome $\mathrm{r}, \mathrm{r}_{1}, \mathrm{r}_{2}$ que são respectivamente os raios de $\mathrm{k}, \mathrm{k}_{1}, \mathrm{k}_{2}$ e também considere $\mathrm{M}$ e $\mathrm{N}$ a interseção $\overleftrightarrow{\mathrm{AC}}$ e $\overleftrightarrow{\mathrm{BC}}$ com o k. Como mostra a figura abaixo.

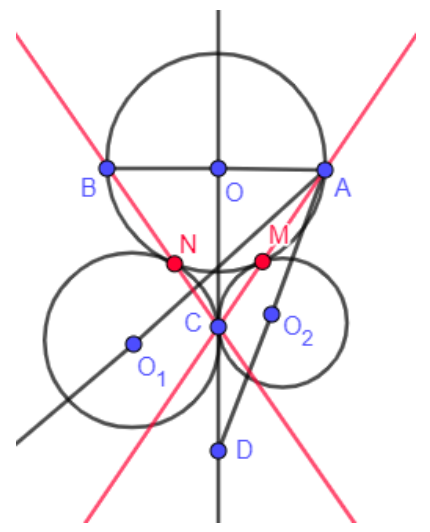

Figura 17: Figura para resolução do exemplo 7. (Fonte: Autores.)

Daí temos que $\triangle \mathrm{AMB}$ é um triângulo retângulo e o triângulo $\Delta \mathrm{AMO}$ é isósceles e

$$
\angle \mathrm{AMO}=\angle \mathrm{OAM}=\angle \mathrm{O}_{1} \hat{\mathrm{CM}}=\angle \mathrm{CMO}_{1} .
$$

Segue que $\mathrm{O}, \mathrm{M}, \mathrm{O}_{1}$ são colineares e

$$
\frac{\overline{\mathrm{AM}}}{\overline{\mathrm{MC}}}=\frac{\overline{\mathrm{OM}}}{\overline{\mathrm{MO}_{1}}}=\frac{\mathrm{r}}{\mathrm{r}_{1}} .
$$


De maneira análoga, $\mathrm{O}, \mathrm{N}, \mathrm{O}_{2}$ são colineares e

$$
\frac{\overline{\mathrm{BN}}}{\overline{\mathrm{NC}}}=\frac{\overline{\mathrm{ON}}}{\overline{\mathrm{NO}_{2}}}=\frac{\mathrm{r}}{\mathrm{r}_{2}} .
$$

Seja P a interseção de l com AB. Os segmentos AN, BM, CP são concorrentes no ortocentro do triângulo $\triangle \mathrm{ABC}$,e, pelo Teorema de Ceva aplicado ao triângulo $\triangle \mathrm{ABC}$, temos

$$
\frac{\overline{\mathrm{AP}}}{\overline{\mathrm{PB}}} \cdot \frac{\overline{\mathrm{CM}}}{\overline{\mathrm{AM}}} \cdot \frac{\overline{\mathrm{NB}}}{\overline{\mathrm{CN}}}=1 \text {, }
$$

de maneira que isolando a primeira razão ficamos com

$$
\frac{\overline{\mathrm{AP}}}{\overline{\mathrm{PB}}}=\frac{\overline{\mathrm{AM}}}{\overline{\mathrm{CM}}} \cdot \frac{\overline{\mathrm{CN}}}{\overline{\mathrm{NB}}}=\frac{\mathrm{r}}{\mathrm{r}_{1}} \cdot \frac{\mathrm{r}_{2}}{\mathrm{r}}=\frac{\mathrm{r}_{2}}{\mathrm{r}_{1}} .
$$

A identidade (20) acima pode ser reescrita como

$$
\frac{\mathrm{r}_{1}}{\overline{\mathrm{PB}}}=\frac{\mathrm{r}_{2}}{\overline{\mathrm{AP}}} \text {. }
$$

Agora tome $\mathrm{D}_{1}$ e $\mathrm{D}_{2}$ as interseções de 1 com $\mathrm{BO}_{1}$ e $\mathrm{AO}_{2}$. Então,

$$
\frac{\overline{\mathrm{CD}_{1}}}{\overline{\overline{\mathrm{D}_{1} \mathrm{P}}}}=\frac{\overline{\mathrm{O}_{1} \mathrm{C}}}{\overline{\mathrm{PB}}}=\frac{\mathrm{r}_{1}}{\overline{\mathrm{PB}}},
$$

e, similarmente,

$$
\frac{\overline{\mathrm{CD}_{2}}}{\overline{\mathrm{D}_{2} \mathrm{P}}}=\frac{\mathrm{r}_{2}}{\overline{\mathrm{PA}}} .
$$

Portanto, temos que

$$
\frac{\overline{\mathrm{CD}_{1}}}{\overline{\mathrm{D}_{1} \mathrm{P}}}=\frac{\overline{\mathrm{CD}_{2}}}{\overline{\mathrm{D}_{2} \mathrm{P}}},
$$

o que nos leva a concluir que $\mathrm{D}_{1}=\mathrm{D}_{2}$. Dessa maneira, $\mathrm{AO}_{2}, \mathrm{BO}_{1}$ e 1 tem o único ponto $\mathrm{D}_{1}=\mathrm{D}_{2}$ em comum.

Finalizaremos esta seção com uma variação do Teorema de Ceva que é bastante utilizada no contexto olímpico, que é a sua forma trigonométrica.

Exemplo 8 (Forma Trigonométrica de Ceva). Dado um triângulo $\triangle \mathrm{ABC}$ qualquer, considere as cevianas AX, BY e CZ, conforme a Figura 18.

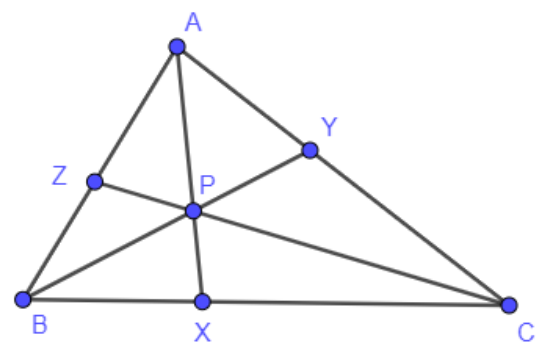

Figura 18: Triângulo com cevianas concorrentes. (Fonte: Autores.) 
As cevianas são concorrentes, se e somente se,

$$
\frac{\operatorname{sen}(\angle \mathrm{ABY})}{\operatorname{sen}(\angle \mathrm{CBY})} \cdot \frac{\operatorname{sen}(\angle \mathrm{B} \hat{\mathrm{C} Z})}{\operatorname{sen}(\angle \mathrm{A} \hat{\mathrm{CZ}})} \cdot \frac{\operatorname{sen}(\angle \mathrm{C} \hat{\mathrm{AX}})}{\operatorname{sen}(\angle \mathrm{B} \hat{\mathrm{AX}})}=1
$$

Demonstração. Primeiramente observe que, pelo Teorema de Ceva, as cevianas são concorrentes se, e somente se,

$$
\frac{\overline{\mathrm{AZ}}}{\overline{\mathrm{BZ}}} \cdot \frac{\overline{\mathrm{BX}}}{\overline{\mathrm{CX}}} \cdot \frac{\overline{\mathrm{CY}}}{\overline{\mathrm{AY}}}=1 \text {. }
$$

Perceba que a identidade anterior é equivalente a

$$
\frac{[\mathrm{APZ}]}{[\mathrm{BPZ}]} \cdot \frac{[\mathrm{BPX}]}{[\mathrm{CPX}]} \cdot \frac{[\mathrm{CPY}]}{[\mathrm{APY}]}=1
$$

Para perceber esse fato, note que os triângulos $\triangle \mathrm{APZ}$ e $\triangle \mathrm{BPZ}$ possuem a mesma altura $\mathrm{h}$ em relação ao vértice $\mathrm{P}$, portanto,

$$
\frac{[\mathrm{APZ}]}{[\mathrm{BPZ}]}=\frac{\overline{\mathrm{AZ}} \cdot \mathrm{h}}{\overline{\mathrm{BZ}} \cdot \mathrm{h}}=\frac{\overline{\mathrm{AZ}}}{\overline{\mathrm{BZ}}},
$$

e de maneira análoga para os outras duas razões. Além disso, usando a fórmula da área de um triângulo que utiliza dois lados e o ângulo entre eles, temos que

$$
[\mathrm{APZ}]=\frac{\overline{\mathrm{AP}} \cdot \overline{\mathrm{AZ}} \cdot \operatorname{sen}(\angle \mathrm{BAX})}{2} \text {; }
$$

de mesmo modo, para as outras áreas, portanto,

$$
\frac{[\mathrm{APZ}]}{[\mathrm{BPZ}]}=\frac{\frac{\overline{\mathrm{AP}} \cdot \overline{\mathrm{AZ}} \cdot \operatorname{sen}(\angle \mathrm{BAX})}{2}}{\frac{\overline{\mathrm{BP}} \cdot \overline{\mathrm{BZ}} \cdot \operatorname{sen}(\angle \mathrm{ABY})}{2}}=\frac{\overline{\mathrm{AP}} \cdot \overline{\mathrm{AZ}} \cdot \operatorname{sen}(\angle \mathrm{BAX})}{\overline{\mathrm{BP}} \cdot \overline{\mathrm{BZ}} \cdot \operatorname{sen}(\angle \mathrm{ABY})} \text {. }
$$

Analogamente para as outras duas razões,

$$
\frac{[\mathrm{BPX}]}{[\mathrm{CPX}]}=\frac{\overline{\mathrm{BP}} \cdot \overline{\mathrm{BX}} \cdot \operatorname{sen}(\angle \mathrm{CBY})}{\overline{\mathrm{CP}} \cdot \overline{\mathrm{CX}} \cdot \operatorname{sen}(\angle \mathrm{BC} Z)} \quad \text { e } \quad \frac{[\mathrm{CPY}]}{[\mathrm{APY}]}=\frac{\overline{\mathrm{CP}} \cdot \overline{\mathrm{CY}} \cdot \operatorname{sen}(\angle \mathrm{A} \hat{\mathrm{CZ}})}{\overline{\mathrm{AP}} \cdot \overline{\mathrm{AY}} \cdot \operatorname{sen}(\angle \mathrm{CAX})} .
$$

Assim,

$$
\begin{aligned}
1 & =\frac{[\mathrm{APZ}]}{[\mathrm{BPZ}]} \cdot \frac{[\mathrm{BPX}]}{[\mathrm{CPX}]} \cdot \frac{[\mathrm{CPY}]}{[\mathrm{APY}]} \\
& =\frac{\overline{\mathrm{AZ}} \cdot \operatorname{sen}(\angle \mathrm{BAX})}{\overline{\mathrm{BZ}} \cdot \operatorname{sen}(\angle \mathrm{ABY})} \cdot \frac{\overline{\mathrm{BX}} \cdot \operatorname{sen}(\angle \mathrm{CBY})}{\overline{\mathrm{CX}} \cdot \operatorname{sen}(\angle \mathrm{B} \hat{\mathrm{CZ}})} \cdot \frac{\overline{\mathrm{CY}} \cdot \operatorname{sen}(\angle \mathrm{AC} Z)}{\overline{\mathrm{AY}} \cdot \operatorname{sen}(\angle \mathrm{CAX})} \\
& =\left(\frac{\overline{\mathrm{AZ}}}{\overline{\mathrm{BZ}}} \cdot \frac{\overline{\mathrm{BX}}}{\overline{\mathrm{CX}}} \cdot \frac{\overline{\mathrm{CY}}}{\overline{\mathrm{AY}}}\right) \cdot \frac{\operatorname{sen}(\angle \mathrm{B} \hat{\mathrm{AX}})}{\operatorname{sen}(\angle \mathrm{ABY})} \cdot \frac{\operatorname{sen}(\angle \mathrm{CBY})}{\operatorname{sen}(\angle \mathrm{B} \hat{\mathrm{CZ}})} \cdot \frac{\operatorname{sen}(\angle \mathrm{A} \hat{\mathrm{CZ}})}{\operatorname{sen}(\angle \mathrm{C} \hat{\mathrm{AX}})} \\
& =\frac{\operatorname{sen}(\angle \mathrm{B} \hat{\mathrm{AX}})}{\operatorname{sen}(\angle \mathrm{ABY})} \cdot \frac{\operatorname{sen}(\angle \mathrm{CBY})}{\operatorname{sen}(\angle \mathrm{BC} Z)} \cdot \frac{\operatorname{sen}(\angle \mathrm{A} \hat{\mathrm{CZ}})}{\operatorname{sen}(\angle \mathrm{CAX})} .
\end{aligned}
$$

Tomando o inverso multiplicativo em cada lado e organizando a expressão acima, obtemos a identidade desejada. 


\section{Representações distintas de um mesmo resultado?}

Já sabemos nesse ponto do trabalho que o resultado do Teorema de Menelaus trata sobre colinearidade de pontos, e o de Ceva aborda concorrência de retas ou segmentos. Ainda assim, a escrita, imagens, hipóteses e teses dos dois teoremas, por serem muito parecidos, induzem-nos a pensar que esses dois resultados podem ser equivalentes, ou seja, do ponto de vista matemático, representam um mesmo resultado. Observe que na primeira implicação da demonstração do Teorema de Ceva, utilizamos o Teorema de Menelaus. É possível mostrar a recíproca, ou seja, assumido o Teorema de Ceva, conseguimos demonstrar o Teorema de Menelaus (ver [10]). Isso nos diz que os Teoremas são equivalentes, respondendo, portanto, a pergunta que nomeia esta seção. Mostraremos, porém, que não obtemos os mesmos objetos quando aplicamos os dois teoremas sob um mesmo triângulo, mas, ainda assim, esses objetos possuem uma determinada ligação.

Definição 2. Sejam A, B, M e N pontos distribuídos em uma reta, todos distintos conforme a disposição da Figura 19

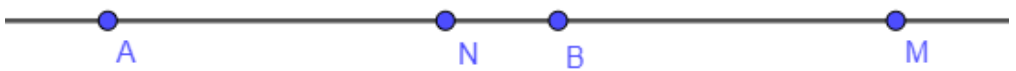

Figura 19: Representação de conjugados harmônicos. (Fonte: Autores.)

Dizemos que os pontos $\mathrm{M}$ e $\mathrm{N}$ dividem harmonicamente o segmento $\mathrm{AB}$ se

$$
\frac{\overline{\mathrm{NA}}}{\overline{\mathrm{NB}}}=\mathrm{k}=\frac{\overline{\mathrm{MA}}}{\overline{\mathrm{MB}}},
$$

ou seja, quando $\mathrm{M}$ e $\mathrm{N}$ dividem o segmento $\mathrm{AB}$ na mesma razão. Nessa situação $\mathrm{M}$ e $\mathrm{N}$ são ditos conjugados harmônicos na razão k sobre AB.

Em posse dessa definição veremos como é possível relacionar os Teoremas de Menelaus e Ceva, respondendo assim o título da seção. Para relacionar esses teoremas iremos resolver o seguinte exemplo.

Exemplo 9 (SIMO - Singapore Mathematical Society - Training Problems/2005). Seja um quadrilátero $\mathrm{CBZY}$ onde $\mathrm{P}$ é o ponto de interseção das retas $\overleftrightarrow{\mathrm{CZ}}$ e $\overleftrightarrow{\mathrm{BY}}$, A é a interseção das retas $\overleftrightarrow{\mathrm{CY}}$ e $\overleftrightarrow{\mathrm{BZ}}, \mathrm{X}_{1}$ é a interseção das retas $\overleftrightarrow{\mathrm{YZ}}$ e $\overleftrightarrow{\mathrm{CB}}$ e X o ponto de interseção das retas $\overleftrightarrow{\mathrm{AP}}$ e $\overleftrightarrow{\mathrm{CB}}$. Prove que $\mathrm{X}$ e $\mathrm{X}_{1}$ são conjugados harmônicos do segmento CB.

Solução. Traduzindo as informações acima em uma figura temos 


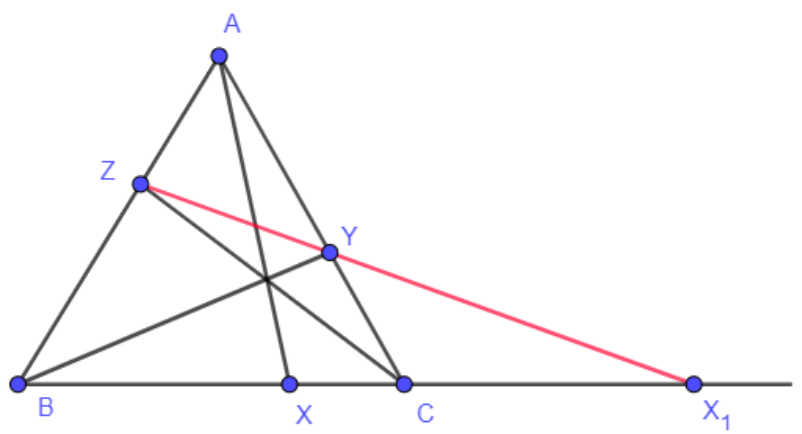

Figura 20: Figura para visualização do exemplo 9. (Fonte: Autores.)

Aplicando o Teorema de Ceva ao triângulo $\triangle \mathrm{ABC}$ com os segmentos CZ, BY e AX, temos:

$$
\frac{\overline{\mathrm{ZA}}}{\overline{\mathrm{ZB}}} \cdot \frac{\overline{\mathrm{BX}}}{\overline{\mathrm{CX}}} \cdot \frac{\overline{\mathrm{CY}}}{\overline{\mathrm{AY}}}=1 .
$$

Pelo Teorema de Menelaus no $\Delta \mathrm{ABC}$ com pontos $\mathrm{Y}, \mathrm{Z}, \mathrm{X}_{1}$, obtemos

$$
\frac{\overline{\mathrm{ZA}}}{\overline{\mathrm{ZB}}} \cdot \frac{\overline{\mathrm{BX}_{1}}}{\overline{\mathrm{CX}_{1}}} \cdot \frac{\overline{\mathrm{CY}}}{\overline{\mathrm{AY}}}=1 \text {. }
$$

Combinando as equações (21) e (22) e simplificando os termos em comum nas expressões, concluímos que

$$
\frac{\overline{\mathrm{BX}}}{\overline{\mathrm{CX}}}=\frac{\overline{\mathrm{BX}_{1}}}{\overline{\mathrm{CX}_{1}}},
$$

e como $\mathrm{X}$ e $\mathrm{X}_{1}$ são pontos distintos eles então são conjugados harmônicos.

Com isso concluímos que embora os Teoremas de Ceva e de Menelaus sejam equivalentes, aplicando os teoremas ao mesmo triângulo, produzimos objetos $\mathrm{X}$ e $\mathrm{X}_{1}$ que não são iguais, mas estes intimamente ligados pois são conjugados harmônicos de razão $\mathrm{k}$ para algum $\mathrm{k} \in \mathbb{R}$ sobre o lado $\mathrm{AB}$ do triângulo $\triangle \mathrm{ABC}$.

\section{Problemas Complementares}

Nesta seção fizemos uma seleção de alguns problemas que podem ser solucionados com as técnicas que foram apresentadas e que consolidam os conhecimentos abordados aqui, para que os leitores interessados possam se desafiar na busca pelas soluções dessas questões olímpicas. Reforçamos que os quatro problemas iniciais complementam as ferramentas apresentadas e abrem possibilidades para novas questões olímpicas. Ressaltamos que as traduções dessas questões foram feitas pelos autores, então podem estar diferentes de outros materiais e adequamos as notações para que os enunciados ficassem condizentes com as notações utilizadas neste trabalho, de modo que não haja confusão de conceitos na interpretação de cada problema.

Problema 1 (Poti - Geometria - Nível 2). Prove que 
1. As medianas de um triângulo são concorrentes em um único ponto (o baricentro).

2. As bissetrizes internas de um triângulo são concorrentes em um único ponto (o incentro).

Problema 2 (O Teorema de Desargues). Utilizando o Teorema de Menelaus, prove que dados dois triângulos $\Delta \mathrm{ABC}$ e $\Delta \mathrm{A}^{\prime} \mathrm{B}^{\prime} \mathrm{C}^{\prime}$, se as retas $\overleftrightarrow{\mathrm{AA}^{\prime}}, \overleftrightarrow{\mathrm{BB}^{\prime}}$ e $\overleftrightarrow{\mathrm{CC}^{\prime}}$ são concorrentes em um único ponto $\mathrm{O}$, então os pontos $\mathrm{P}=\overleftrightarrow{\mathrm{BC}} \cap \overleftrightarrow{\mathrm{B}^{\prime} \mathrm{C}^{\prime}}, \mathrm{Q}=\overleftrightarrow{\mathrm{CA}} \cap \overleftrightarrow{\mathrm{C}^{\prime} \mathrm{A}^{\prime}}$ e $\mathrm{R}=\overleftrightarrow{\mathrm{AB}} \cap \overleftrightarrow{\mathrm{A}^{\prime} \mathrm{B}^{\prime}}$ são colineares.

Problema 3 (O Teorema de Pappus). Utilizando o Teorema de Menelaus prove que se $A_{1}, A_{2}$ e $A_{2}$ são colineares e $B_{1}, B_{2}$ e $B_{3}$ também são colineares, então os pontos de interseção $A_{1} B_{2} \cap A_{2} B_{1}$, $\mathrm{A}_{1} \mathrm{~B}_{3} \cap \mathrm{A}_{3} \mathrm{~B}_{1}$ e $\mathrm{A}_{2} \mathrm{~B}_{3} \cap \mathrm{A}_{3} \mathrm{~B}_{2}$ são colineares.

Problema 4 (Conjugado Isogonal do Ponto de Gergonne). Dado um triângulo $\triangle \mathrm{ABC}$ e sejam $\mathrm{D}$, $\mathrm{E}, \mathrm{F}$ os pés das alturas relativas aos vértices $\mathrm{A}, \mathrm{B}$ e $\mathrm{C}$ respectivamente, construa as circunferências inscritas aos triângulos $\triangle \mathrm{AEF}, \triangle \mathrm{BDF}$ e $\triangle \mathrm{CDE}$, e denote os pontos de tangência com os segmentos $\mathrm{DE}, \mathrm{EF}$ e FD por $\mathrm{C}^{\prime}, \mathrm{A}^{\prime}$ e $\mathrm{B}^{\prime}$ respectivamente. Prove que os segmentos $\mathrm{AA}^{\prime}, \mathrm{BB}^{\prime}$ e $\mathrm{CC}^{\prime}$ são concorrentes. (Dica: Use a forma trigonométrica do Teorema de Ceva.)

Problema 5 (IMO/1998 - Hong Kong Preliminary Selection Contest). Dado um triângulo $\triangle \mathrm{ABC}$, sejam $\mathrm{E}, \mathrm{F}$ e $\mathrm{G}$ pontos sobre os lados $\mathrm{AB}, \mathrm{BC}$ e $\mathrm{CA}$, respectivamente e tais que

$$
\frac{\overline{\mathrm{AE}}}{\overline{\mathrm{EB}}}=\frac{\overline{\mathrm{BF}}}{\overline{\overline{\mathrm{FC}}}}=\frac{\overline{\mathrm{CG}}}{\overline{\mathrm{GA}}}=\frac{1}{3} \text {. }
$$

Sejam $K=\overleftrightarrow{\mathrm{AF}} \cap \overleftrightarrow{\mathrm{CE}}, \mathrm{L}=\overleftrightarrow{\mathrm{BG}} \cap \overleftrightarrow{\mathrm{AF}}$ e $M=\overleftrightarrow{\mathrm{CE}} \cap \overleftrightarrow{\mathrm{BG}}$. Supondo que a área do triângulo $\triangle \mathrm{ABC}$ seja 1 , encontre a área do triângulo $\triangle \mathrm{KLM}$.

Problema 6 ( APMO/1989 - Asian Pacific Mathematics Olympiad). Dados $\mathrm{A}_{1}, \mathrm{~A}_{2}$ e $\mathrm{A}_{3}$ três pontos não colineares no plano, e por conveniência considere $A_{4}=A_{1}$ e $A_{5}=A_{2}$. Para $n=1,2$ e 3 , sejam $B_{n}$ e $C_{n}$ os pontos médios de $A_{n} A_{n+1}$ e $A_{n} B_{n}$, respectivamente, e $D_{n}=A_{n} C_{n+1} \cap B_{n} A_{n+2}$ e $\mathrm{E}_{\mathrm{n}}=\mathrm{A}_{\mathrm{n}} \mathrm{B}_{\mathrm{n}+1} \cap \mathrm{C}_{\mathrm{n}} \mathrm{A}_{\mathrm{n}+2}$. Calcule a razão das medidas de área $\left[\mathrm{D}_{1} \mathrm{D}_{2} \mathrm{D}_{3}\right] /\left[\mathrm{E}_{1} \mathrm{E}_{2} \mathrm{E}_{3}\right]$.

Problema 7 (Austrália/1995). As retas unindo os três vértices do triângulo $\triangle \mathrm{ABC}$ a um ponto nesse plano corta os lados opostos aos vértices $\mathrm{A}, \mathrm{B}, \mathrm{C}$ nos pontos $\mathrm{K}, \mathrm{L}, \mathrm{M}$, respectivamente. Uma reta contendo o ponto $\mathrm{M}$ paralelo a $\mathrm{KL}$ corta $\mathrm{BC}$ em V e AK em W. Prove que $\overline{\mathrm{VM}}=\overline{\mathrm{MW}}$.

Problema 8 (Olimpíada do Cone Sul). Seja C uma circunferência de centro O, AB um diâmetro de $\mathrm{C}$ e $\mathrm{R}$ um ponto qualquer em $\mathrm{C}$ distinto de $\mathrm{A}$ e de B. Seja $\mathrm{P}$ a interseção da perpendicular traçada por $\mathrm{O}$ a AR. Sobre a reta $\overleftrightarrow{\mathrm{OP}}$ marca-se o ponto $\mathrm{Q}$, de maneira que $\overrightarrow{\mathrm{QP}}$ é a metade de $\overline{\mathrm{PO}}$ e $Q$ não pertence ao segmento $\mathrm{OP}$. Por $\mathrm{Q}$ traçamos a paralela a $\mathrm{AB}$ que corta a reta $\overleftrightarrow{\mathrm{AR}} \mathrm{em} T$. Chamamos de $\mathrm{H}$ o ponto de interseção das retas $\overleftrightarrow{\mathrm{AQ}}$ e $\overleftrightarrow{\mathrm{OT}}$. Prove que $\mathrm{H}, \mathrm{R}$ e $\mathrm{B}$ são colineares. (Dica: Use o Teorema de Menelaus.)

Problema 9 (Canadá/1994). Seja $\triangle \mathrm{ABC}$ um triângulo acutângulo. Seja AD a altura relativa ao lado $\mathrm{BC}$ e $\mathrm{H}$ um ponto qualquer sobre o segmento $\mathrm{AD}$, de forma que as retas $\overleftrightarrow{\mathrm{BH}}$ e $\overleftrightarrow{\mathrm{CH}}$ intersectam $\mathrm{AC}$ e $\mathrm{AB}$ em E e F, respectivamente. Prove que $\angle \mathrm{ED} H=\angle \mathrm{FD} H$.

Problema 10 (Reino Unido/1998). No triângulo $\triangle \mathrm{ABC}$, D é o ponto médio de $\mathrm{AB}$ e $\mathrm{E}$ é um ponto sobre o lado $\mathrm{BC}$ tal que $\overline{\mathrm{BE}}=2 \cdot \overline{\mathrm{EC}}$. Sabendo que $\angle \mathrm{A} \mathrm{BC}=\angle \mathrm{BRE}$, calcule o valor de $\angle \mathrm{BRC}$.

Problema 11 (Korea/1997-1988). Em um triângulo agudo $\triangle \mathrm{ABC}$ com $\overline{\mathrm{AB}} \neq \overline{\mathrm{AC}}$, seja $\mathrm{V}$ a intersecção da bissetriz do ângulo $\angle \mathrm{BA} C$ e seja $\mathrm{D}$ o pé da altura relativa ao vértice $\mathrm{A}$. Se $\mathrm{E}$ e $\mathrm{F}$ são as intersecções dos círculos circunscritos aos triângulos $\triangle \mathrm{AVD}$ com $\mathrm{CA}$ e $\mathrm{AB}$, respectivamente, mostre que $\mathrm{AD}, \mathrm{BE}$ e CF são concorrentes. (Dica: Utilize o Teorema de Ceva.) 
Problema 12 (Tchecoslováquia/1999). Dado um triângulo $\Delta \mathrm{ABC}$, considere pontos K, L e M no interior dos lados $\mathrm{BC}, \mathrm{CA}, \mathrm{AB}$, respectivamente, tais que os segmentos $\mathrm{AK}, \mathrm{BL}$ e CM intersectemse em um ponto $\mathrm{U}$. Prove que se os triângulos $\triangle \mathrm{AMU}$ e $\Delta \mathrm{KCU}$ têm medida de área $\mathrm{P}$ e os triângulos $\triangle \mathrm{MBU}$ e $\Delta \mathrm{CLU}$ têm a medida de área $\mathrm{Q}$, então $\mathrm{P}=\mathrm{Q}$.

Problema 13 (Zeitz/96). Seja ABCDEF um hexágono convexo (dados dois pontos no hexágono, o segmento definido por esses pontos está inteiramente contido no polígono) e circunscritível. Prove que $\mathrm{AD}, \mathrm{BE} \mathrm{CF}$ são concorrentes se, e somente se, $\overline{\mathrm{AB}} \cdot \overline{\mathrm{CD}} \cdot \overline{\mathrm{EF}}=\overline{\mathrm{BC}} \cdot \overline{\mathrm{DE}} \cdot \overline{\mathrm{FA}}$. (Dica: Use a forma trigonométrica do Teorema de Ceva).

\section{Referências}

[1] BEZERRA, Yury. Geometria Projetiva: algumas aplicações básicas para alunos do Ensino Médio. 2014. Dissertação de Profmat-Ufam, Amazonas, 2014.

[2] CHAVES, A. P. A. "Álgebra e teoria dos números para olimpíadas." Professor de Matemática On-line (PMO), v. 7, $\mathrm{n}^{\mathrm{O}}$ 1, p. 66-76, 2019.

[3] COSTA, Thafarel. O princípio da dualidade na geometria de Galileu. 2018. Dissertação de Profmat-UnB, Brasília, 2018.

[4] FERREIRA. Francisco. Unificando os teoremas de Menelaus e Ceva. 2017. Dissertação de Profmat-UFCA, Ceará, 2017.

[5] International Mathematical Olympiad. Disponível em: <https://www.imo-official.org/>. Acesso em 28 Jan. de 2021.

[6] LÓPEZ, J. ; BRUNO-ALFOnSO, A. ; BARBOSA, G. F. "Bases numéricas na olimpíada internacional de matemática." Professor de Matemática On-line (PMO), v. 7, n² 2, p. 195-204, 2019 .

[7] Math Olympiad teaching notes em: <https://www.math.cmu.edu/ ploh/olympiad.shtml>. Acesso em 2 Jan. de 2021.

[8] MIENTKA, walter. Mathematical Olympiads (1995 - 1996) - Olympiads Problems Around the World. IMO Advisory Broad.

[9] Olimpíada Brasileira de Matemática. Disponível em: <https://www.obm.org.br/>. Acesso em 29 Jan. de 2021.

[10] Pollard, J. "Ceva = (Menelaus) ${ }^{2}$." The Mathematical Gazette, v. 84, nº 500, p. 268-271. 2000.

[11] Polos Olímpicos de Treinamento Intensivo. Disponível em: <https://poti.impa.br/>. Acesso em 28 Jan. de 2021.

[12] School of Mathematics and Statistics University of St Andrews, Scotland MacTutor History of Mathematics archive, 2020. Disponível em: <https://mathshistory.st-andrews.ac.uk/ Biographies/>. Acesso em 10 Ago. de 2020.

[13] Seleção Cone Sul. Disponível em: <https://sites.google.com/site/selecaoconesul/>. Acesso em 28 Jan. de 2021.

[14] SILVA, José. Os teoremas de Menelaus e Ceva. 2015. Dissertação de Profmat-UFRPE, Recife, 2015.

[15] The Korean Mathematical Olympiad. Disponível em: <http://www.kmo.or.kr/main.html>. Acesso em 28 Jan. de 2021. 
Jhonata Avelar dos Santos Universidade Federal Rural de Pernambuco Departamento de Matemática (DM-UFRPE) Recife/PE $<$ jhonata.avelar@hotmail.com>

Pedro Victor S. Freitas Universidade Federal Rural de Pernambuco Departamento de Matemática (DM-UFRPE) Recife/PE $<$ pedro-victor@msn.com>

Gilson S. Ferreira Jr. Universidade Federal Rural de Pernambuco Departamento de Matemática (DM-UFRPE) Recife/PE $<$ gilson.simoesj@ufrpe.br>

Thiago Yukio Tanaka Universidade Federal Rural de Pernambuco Departamento de Matemática (DM-UFRPE) Recife/PE $<$ thiago.tanaka@ufrpe.br>

Recebido: $03 / 04 / 2021$ Publicado: 24/06/2021 